Rodriguez-Ferreiro, J, Gennari, S P, Davies, R and Cuetos, F

Neural correlates of abstract verb processing.

Rodriguez-Ferreiro, J, Gennari, S P, Davies, R and Cuetos, F (2011) Neural correlates of abstract verb processing. Journal of Cognitive Neuroscience , 23 (1). pp. 106-118.

Doi:10.1162/jocn.2010.21414

This version is available https://radar.brookes.ac.uk/radar/items/f2570140-a8aa-1923-9745-be763dc985c0/1/

Available on RADAR: September 2012

Copyright (C and Moral Rights are retained by the author(s) and/ or other copyright owners. A copy can be downloaded for personal non-commercial research or study, without prior permission or charge. This item cannot be reproduced or quoted extensively from without first obtaining permission in writing from the copyright holder(s). The content must not be changed in any way or sold commercially in any format or medium without the formal permission of the copyright holders.

This document is the published version] of the journal article. Some differences between the published version and this version may remain and you are advised to consult the published version if you wish to cite from it. 


\title{
Neural Correlates of Abstract Verb Processing
}

\author{
Javier Rodríguez-Ferreiro ${ }^{1}$, Silvia P. Gennari ${ }^{2}$, Robert Davies ${ }^{3}$, \\ and Fernando Cuetos ${ }^{4}$
}

\begin{abstract}
The present study investigated the neural correlates of the processing of abstract (low imageability) verbs. An extensive body of literature has investigated concrete versus abstract nouns but little is known about how abstract verbs are processed. Spanish abstract verbs including emotion verbs (e.g., amar, "to love"; molestar, "to annoy") were compared to concrete verbs (e.g., llevar, "to carry"; arrastrar, "to drag"). Results indicated that abstract verbs elicited stronger activity in regions previously associated with semantic retrieval such as inferior frontal, anterior temporal, and posterior temporal regions, and that concrete and abstract activation networks (compared to that of pseudoverbs) were partially distinct, with concrete verbs eliciting more posterior activity in these regions. In contrast to previous studies
\end{abstract}

\section{INTRODUCTION}

Several studies have demonstrated that understanding concrete words involves activation of representations of the sensory-motor experience associated with their meanings. Action verbs, in particular, have been shown to recruit distributed neural networks that include regions close to, or overlapping with, the areas involved in the perception, programming, and execution of those actions (Kemmerer, González-Castillo, Talavage, Patterson, \& Wiley, 2007; Tettamanti et al., 2005; Hauk, Johnsrude, \& Pulvermüller, 2004). Motion verbs such as running, for example, engage posterior temporal regions near those involved in the encoding of motion (Kable, Lease-Spellmeyer, \& Chatterjee, 2002; Grezes \& Decety, 2001; Martin \& Chao, 2001). A great proportion of verbs, however, do not refer to physical actions and lack obvious sensory-motor associations. Verbs such as believe, adore, or solve, for example, do not seem to have strong associations with physical actions in the way that running does. How do we process and represent the meaning of such verbs?

Theories of concrete and abstract words have often been cast in representational terms. The dual-coding theory, for example, suggests that, although concrete semantics can be represented in both sensory-based and verbal-symbolic codes, abstract words are primarily encoded verbally in the

${ }^{1}$ Universitat de Barcelona, Spain, ${ }^{2}$ University of York, UK, ${ }^{3}$ Oxford Brookes University, UK, ${ }^{4}$ Universidad de Oviedo, Spain investigating nouns, verbs strongly engage both left and right inferior frontal gyri, suggesting, as previously found, that right prefrontal cortex aids difficult semantic retrieval. Together with previous evidence demonstrating nonverbal conceptual roles for the active regions as well as experiential content for abstract word meanings, our results suggest that abstract verbs impose greater demands on semantic retrieval or property integration, and are less consistent with the view that abstract words recruit left-lateralized regions because they activate verbal codes or context, as claimed by proponents of the dual-code theory. Moreover, our results are consistent with distributed accounts of semantic memory because distributed networks may coexist with varying retrieval demands.

left hemisphere (Sadoski \& Paivio, 2004; Paivio, 1986, 1991) and emotion words may elicit nonverbal emotion-related activation (cf. Paivio, 1986, pp. 271-272). Barsalou (1999, 2008), Barsalou and Wiemer-Hastings (2005), and WiemerHastings and $\mathrm{Xu}$ (2005), in contrast, have proposed that all concepts are experiential and grounded in modalityspecific knowledge that reflects the situations in which their referents typically occur, although different concepts are centered on different aspects of those situations. Concrete concepts are grounded in sensory-motor features, whereas abstract concepts are grounded in multiple types of information such as interpersonal events, sensory-motor features, or the introspective experiences associated with them.

It is difficult, however, to infer from observed behavior or neural activity how abstract words are represented because, although the results can be interpreted in representational terms, an alternative account can be offered in terms of the relative difficulty of retrieval or integration of semantic features for abstract compared to concrete verbs. Many neuroimaging studies in the concrete-abstract domain have assumed that observed neural activity reflects the activation of semantic representations (Ghio \& Tettamanti, 2010; Pexman, Hargreaves, Edwards, Henry, \& Goodyear, 2007b; Binder, Westbury, McKiernan, Possing, \& Medler, 2005; Sabsevitz, Medler, Seidenberg, \& Binder, 2005; Noppeney \& Price, 2004). In particular, higher activity for abstract words in left-lateralized language regions has been taken to support the dual-coding theory, as putative 
verbal codes would be activated there. Nevertheless, activity in some of those regions, such as the left inferior frontal gyrus (LIFG), has typically been attributed to integrative, regulatory, or retrieval functions rather than to the activation of knowledge representation (e.g., ThompsonSchill, Bedny, \& Goldberg, 2005; Wagner, Paré-Blagoev, Clark, \& Poldrack, 2001). Differential activity in such regions for concrete and abstract words may indicate, from this alternative perspective, that concrete and abstract words differ in retrieval processes and that abstract words require more controlled or effortful integration due to their less imageable representations, rather than less imageable representations being specifically stored or located in such regions.

The difficulty in understanding abstract concepts is exacerbated by the fact that neuroimaging studies investigating concrete versus abstract words have not converged on the same regions of brain activation, and do not always share theoretical perspectives. Although some studies investigating nouns report similar activity in the LIFG and the anterior temporal region (Sabsevitz et al., 2005; Noppeney \& Price, 2004; Perani, Cappa, et al., 1999), many additional activation clusters (up to 25 in one of these studies) have been reported in the right and left hemispheres in these and other studies (Pexman et al., 2007b; Grossman et al., 2002; Kiehl et al., 1999; Mellet, Tzourio, Denis, \& Mazoyer, 1998; D'Esposito et al., 1997). Proposed theoretical explanations have varied greatly, ranging from some variants of the dual-code theory (e.g., Binder et al., 2005; Sabsevitz et al., 2005) to embodied distributed approaches (Pexman et al., 2007b), with some also including processing considerations (e.g., Grossman et al., 2002; for summary of previous noun-related findings, see Pexman et al., 2007b; Sabsevitz et al., 2005).

The few studies that have investigated concrete and abstract verbs have not fared better. Some studies showed no significant activations associated with cognition verbs (e.g., believe) compared to motion verbs, particularly in targeted posterior temporal regions (Bedny, Caramazza, Grossman, Pascual-Leone, \& Saxe, 2008; Rüschemeyer, Brass, \& Friederici, 2007), although in another study, increased verb imageability was correlated with increased activity in this region and in the LIFG (Bedny \& ThomsonSchill, 2006). In contrast, Grossman et al. (2002) reported higher activity for cognition verbs compared to motion verbs in the posterolateral middle temporal gyrus near a region typically associated with motion and/or action information in other studies (Gennari, MacDonald, Postle, \& Seidenberg, 2007; Kemmerer et al., 2007; Kable, Kan, Wilson, Thomson-Schill, \& Chatterjee, 2005; Kable et al., 2002).

In the present study, we compared several types of verbs that vary in imageability. Testing was conducted in Spanish - a language in which grammatical class ambiguities are not present because verbs are clearly marked by their endings (e.g., cantar, "to sing"; vender, "to sell"; cubrir, "to cover"). Half of the verbs referred to highly imageable motion actions (concrete verbs) and the other half referred to less imageable psychological events (abstract verbs). This latter stimulus category was composed of equal numbers of cognitive verbs such as to think and to reason, which occur with sentential complements, and transitive emotion verbs such as to adore or to surprise. The stimulus category motion verbs contained equal numbers of transitive and intransitive motion verbs, so that comparisons across transitive motion and transitive emotion verbs could be conducted, thereby controlling for effects of argument structure demonstrated in behavioral studies (e.g., Shapiro, Nagel, \& Levine, 1993). We used a task that required participants to retrieve the verbs' semantic properties by answering occasional semantic questions after the word had been presented in $20 \%$ of all trails (catch trials). In fMRI block designs, this minimizes the effect of catch trails on overall activity (which is averaged within and across blocks of stimuli), such that activation patterns mostly reflect word reading, rather than question answering. Thus, semantic information was retrieved but comparisons between word meanings or semantic decisions to every word, which were used in previous studies (Bedny et al., 2008; Sabsevitz et al., 2005; Noppeney \& Price, 2004; Grossman et al., 2002), were not required. This is important because attention to specific aspects of a word's meaning (e.g., pleasantness, consumability, or similarity judgments), and not semantic retrieval per se, may explain some of the inconsistencies in the literature, and furthermore, may introduce additional processes (e.g., comparisons) that are not present in simple word reading, making the interpretation of results difficult. Nevertheless, to compare our results with previous findings, we conducted a secondary behavioral experiment to demonstrate that our concrete and abstract verbs do indeed differ in processing time when a decision is required.

We hypothesized that less imageable words are more difficult to retrieve and, therefore, would engage a network of brain regions previously associated with semantic retrieval, such as the LIFG, the posterior middle temporal gyrus (PMTG), and the anterior temporal region (e.g., Pobric, Jefferies, \& Lambon Ralph, 2007; Tyler et al., 2003; Shapiro, Pascual-Leone, Mottaghy, Gangitano, \& Caramazza, 2001; Mummery et al., 1999). We also hypothesized that if retrieval demands are higher for abstract words, additional right hemisphere homologous regions, such as the right IFG, would be recruited to aid their processing, as previous findings have shown right IFG engagement with higher retrieval demands (Wagner et al., 2001; Thompson-Schill, D’Esposito, Aguirre, \& Farah, 1997; see Wagner \& Smith, 2003 for a review). This difficulty-of-retrieval hypothesis is consistent with behavioral studies indicating that abstract words are less supported by associated knowledge: Abstract words are weakly associated with other concepts (Schwanenflugel, 1991), have fewer number of associates, and are attributed fewer predicates than concrete words (de Groot, 1989; Jones, 1985) and also appear in more diverse contexts (Audet \& Burgees, 1999). These features of abstract semantic concepts suggest that their meanings 
or semantic features are more difficult to access or retrieve than that of concrete concepts.

\section{METHODS}

\section{Materials}

Two main verb categories were used in this experiment: the concrete verbs category, composed of 40 intransitive and 40 transitive motion verbs (e.g., correr, "to run"; acarrear, "to carry"), and the abstract verbs category, composed of 40 transitive emotion verbs (adorar, irritar, "to adore, to annoy"), which referred to emotional states and 40 cognitive verbs (pensar, "to think"; sospechar, "to suspect"; solucionar, "to solve"), which referred to mental activities. There were about 10 reflexive verbs in our stimulus set that were presented with the reflexive suffix -se (e.g., acuclillarse, "to squat") because the suffix is obligatory. Although Spanish -se is a reflexive suffix, while some verbs, particularly motion verbs, are lexically specified to occur with it, the verb root + suffix is not morphologically productive but, rather, is the only form in which the verbs can appear. Examples of such verbs are escabullirse, "to scurry off"; zambullirse, "to dive"; and acurrucarse, "to crouch," which do not have nonpronominal forms.

Stimuli were matched for log-transformed lexical frequency (Alameda \& Cuetos, 1995); letter, syllable, and phoneme length; as well as mean log-transformed bigram frequency (Davis \& Perea, 2005) across all conditions (all $p s>.1)$. To check that the verbs indeed differ in their abstractness, we collected imageability ratings with an independent set of 10 Spanish native speakers matching the experimental participants. We asked these participants to indicate how easy it was for them to elicit the image of the action represented by the word. Concrete and abstract verbs were significantly different $[t(158)=$ $-13.974, p<.001$ ] on rated imageability. A summary of the stimulus characteristics is presented in Table 1. Transitivity for each verb was determined by establishing the most frequent usage of 20 random corpus searches for the verbs. The baseline condition stimuli consisted of 40 verb-like Spanish legal pseudoverbs that were constructed so as to be word-like, with forms that were pronounceable and orthotactically legal in Spanish, and to be verb-like, with endings that matched one of the three possible verb endings in Spanish, $-a r,-e r$, or $-i r$. The pseudoverbs were matched for length with the experimental items.

\section{Behavioral Pretest}

To check that our stimulus verbs differ in processing times, as suggested by previous findings in the literature, we conducted a lexical decision task with our stimulus materials. The same words and pseudowords used in the main experiment were included in this task. Fifteen native Spanish speakers studying at the University of Oviedo participated in this test and were instructed to indicate whether the visually presented words were real Spanish words. Mean and standard deviations from this task are reported in Table 1. Concrete verbs elicited faster responses than abstract verbs $\left[t_{1}(14)=2.580, p=.022 ; t_{2}(158)=5.548\right.$, $p<.001]$, as did concrete transitive verbs compared to abstract transitive verbs $\left[t_{1}(14)=3.094, p=.008 ; t_{2}(78)=\right.$ $4.576, p<.001]$.

\section{Experimental Protocol}

Stimuli were presented in a series of blocks (10 verbs or pseudoverbs per block) pseudorandomly arranged, with 15-sec rest intervals between blocks. Each block lasted 24 sec. Within a block, each verb was presented in infinitival form (which invariably indicates a verb meaning) for 1500 msec. A cross then appeared for $500 \mathrm{msec}$ before the next verb. The whole set of stimuli was presented twice. Participants were asked to silently read the verbs. Each block included two pseudorandomly interspersed catch trials asking a yes/no question about the meaning of the previously presented verb. These questions referred to

Table 1. Summary of Item Characteristics

\begin{tabular}{lccccc}
\hline & Abstract & Motion & Transitive Emotion & Transitive Motion & Pseudoverbs \\
\hline$n$ & 80 & 80 & 40 & 40 & 40 \\
Log Frequency $\bar{x}(\sigma)$ & $1.70(0.41)$ & $1.64(0.52)$ & $1.65(0.31)$ & $1.7(0.44)$ & - \\
Imageability $\bar{x}(\sigma)$ & $2.81(1.10)$ & $5.53(1.19)$ & $3.17(1.14)$ & $5.23(1.11)$ & $3.15(0.53)$ \\
Syllables $\bar{x}(\sigma)$ & $3.1(0.56)$ & $3.06(0.88)$ & $2.9(0.63)$ & $3.02(0.73)$ \\
Letters $\bar{x}(\sigma)$ & $7.85(1.56)$ & $7.79(1.85)$ & $7.78(1.75)$ & $7.5(1.45)$ & $7.75(1.9)$ \\
Phonemes $\bar{x}(\sigma)$ & $7.8(1.67)$ & $7.51(1.72)$ & $7.7(1.76)$ & $7.25(1.43)$ & $7.7(1.88)$ \\
Bigram LogFreq. $\bar{x}(\sigma)$ & $2.39(0.29)$ & $2.29(0.4)$ & $2.38(0.3)$ & $2.38(0.31)$ & $2.3(0.38)$ \\
Lexical Decision Reaction Times $\bar{x}(\sigma)$ & $607(45.9)$ & $583(54.3)$ & $607(40.5)$ & $579(55.9)$ & $637(73.1)$ \\
\hline
\end{tabular}


typical participants, patients, or agents, of the situations associated with the verbs, for example, for gatear ("to crawl" and adivinar ("to foresee/guess"), the questions were ¿el bebé? ("the baby?") and el futuro ("the future"), respectively, which required a positive answer; or for pellizcar ("to pinch") and irritar ("to annoy"), the questions were ¿con las orejas? ("with the ears?") and la felicidad? ("the happiness"), which required a negative answer. Participants were instructed to indicate whether the question matched the meaning of the previously seen verb by pressing a button with their right hand. Catch trial questions were constructed so that equal numbers of yes and no responses were expected throughout the experiment. For pseudoverbs, the question referred to the number of syllables in the pseudoword. Questions were answered correctly (mean 88\%), indicating that participants paid attention to the task, and, by extension, that the participants were engaged in semantic processing of the verbs. No significant differences between conditions were detected in question performance $\left[F_{1}(4,52)=1.369, p=.257 ; F_{2}(4\right.$, 75) $=1.078, p=.373]$.

\section{MR Data Acquisition}

The experiment was conducted in the York Neuroimaging Center. Fourteen right-handed native Spanish speakers (8 men), aged between 23 and 35 years (average $=29$ years), participated in the experiment. Written informed consent was obtained from all participants. All were paid for their participation in the study. Whole-brain images were acquired with a 3-Tesla magnetic resonance imaging system (GE Signa HD Excite) with an eight-channel head coil (GE Signa Excite 3.0T, High Resolution Brain Array; MRI Devices, Gainesville, FL). Sagittal images covering the whole brain were acquired for functional and structural scans. For functional imaging, EPI images were acquired using a $\mathrm{T} 2 *$-weighted gradient-echo sequence $(\mathrm{TR}=3 \mathrm{sec}, \mathrm{TE}=$ $40 \mathrm{msec}$, acquisition matrix $96 \times 96, \mathrm{FOV}=29 \mathrm{~cm}$, inplane resolution $=3 \times 3 \mathrm{~mm}$, contiguous slice thickness $=$ $4.5 \mathrm{~mm}$ ). After the functional scan, high-resolution T1weighted structural images were acquired using an inversion recovery)-prepared 3-D FSPGR (Fast Spoiled Gradient Echo) pulse sequence ( $\mathrm{TR}=7.5 \mathrm{sec}$, TE $=3 \mathrm{msec}$, acquisition matrix $=256 \times 224$ interpolated to $512 \times 512, \mathrm{FOV}=$ $260 \mathrm{~mm}$, in-plane resolution $=0.5 \times 0.5 \mathrm{~mm}$, slice thickness $=2.6 \mathrm{~mm}$ with an overlap of $1.3 \mathrm{~mm}$ ).

\section{Data Analysis}

First-level and higher-level analyses were separately carried out using FEAT (FMRI Expert Analysis Tool) Version 5.63, part of FSL [Oxford Centre for Functional MRI of the Brain (FMRIB) Software Library; www.fmrib.ox.ac.uk/fsl]. The following processing was applied to the fMRI signal data prior to inferential statistical analysis: motion correction (Jenkinson, Bannister, Brady, \& Smith, 2002); slice-timing correction using Fourier-space time-series phase-shifting; spatial smoothing using a Gaussian kernel of FWHM 8 mm; mean-based intensity normalization of all volumes by the same factor; and high-pass temporal filtering (Gaussianweighted least-squares straight line fitting, with sigma = $50.0 \mathrm{sec}$ ). Time-series statistical analysis was carried out with local autocorrelation correction (Woolrich, Ripley, Brady, \& Smith, 2001). Registration to high-resolution and standard images was carried out using FMRIB's linear registration tool (Jenkinson et al., 2002; Jenkinson \& Smith, 2001). Time series were modeled with covariates representing the block structure convolved with a hemodynamic response function (gamma function). Several contrasts were computed comparing concrete and abstract verbs with the pseudoverb baseline, all concrete with all abstract words, and also transitive emotion with transitive motion verbs. Hypotheses were directly tested within predefined regions of interest (ROIs), and all ROI analyses reported below were cluster-corrected for multiple comparisons $(Z=2.3$, $p=.05)$. However, to give a picture of the overall pattern found across the brain, we report an uncorrected group analysis $(p=.001)$ for comparison to other studies. None of our critical conclusion relies on this uncorrected analysis and these results are reported here simply for reference purposes.

\section{Regions of Interest}

ROI analyses were conducted based on previously reported regions associated with abstract words processing: the LIFG, the left anterior temporal pole (LATP), and the left LPMTG. The first two regions have been consistently associated with the processing of abstract noun semantics in tasks that required retrieval of unspecific semantic knowledge (Binder et al., 2005; Sabsevitz et al., 2005; Noppeney \& Price, 2004; Perani, Schnur, et al., 1999). PMTG has been less consistently activated for abstract words but it has been claimed in numerous studies to be involved in the retrieval of verb semantics (Bedny et al., 2008; Gennari et al., 2007; Tranel, Kemmerer, Adolphs, Damasio, \& Damasio, 2003). A fourth region, the right inferior frontal gyrus (RIFG), was also included on the basis of previous findings indicating that conditions with higher retrieval demands also engage this region (Wagner \& Smith, 2003; Wagner et al., 2001). ROIs were taken from the HarvardOxford Cortical Structure Atlas built into FSL. This atlas provides probability maps of regions in the MNI brain categorized in anatomical terms. We took the regions labeled pars triangularis and pars opercularis for the LIFG and RIFG; left middle temporal gyrus-posterior division; and left temporal pole (see Figure 1 for ROI masks). ROI analyses were carried out separately for each region using FMRIB's Local Analysis of Mixed Effects (Woolrich, Behrens, Beckmann, Jenkinson, \& Smith, 2004; Beckmann, Jenkinson, \& Smith, 2003). Each mixed effect group analysis was conducted within the voxels defined by the anatomical regions above and was cluster-corrected for multiple comparisons with $Z=2.3$ and $p=.05$. The same contrasts 
Figure 1. Top: View of the ROI masks used in the ROI analyses. Bottom: Motion and abstract verbs compared to pseudoverbs and with each other (cluster-corrected $Z=2.3, p=.05)$.

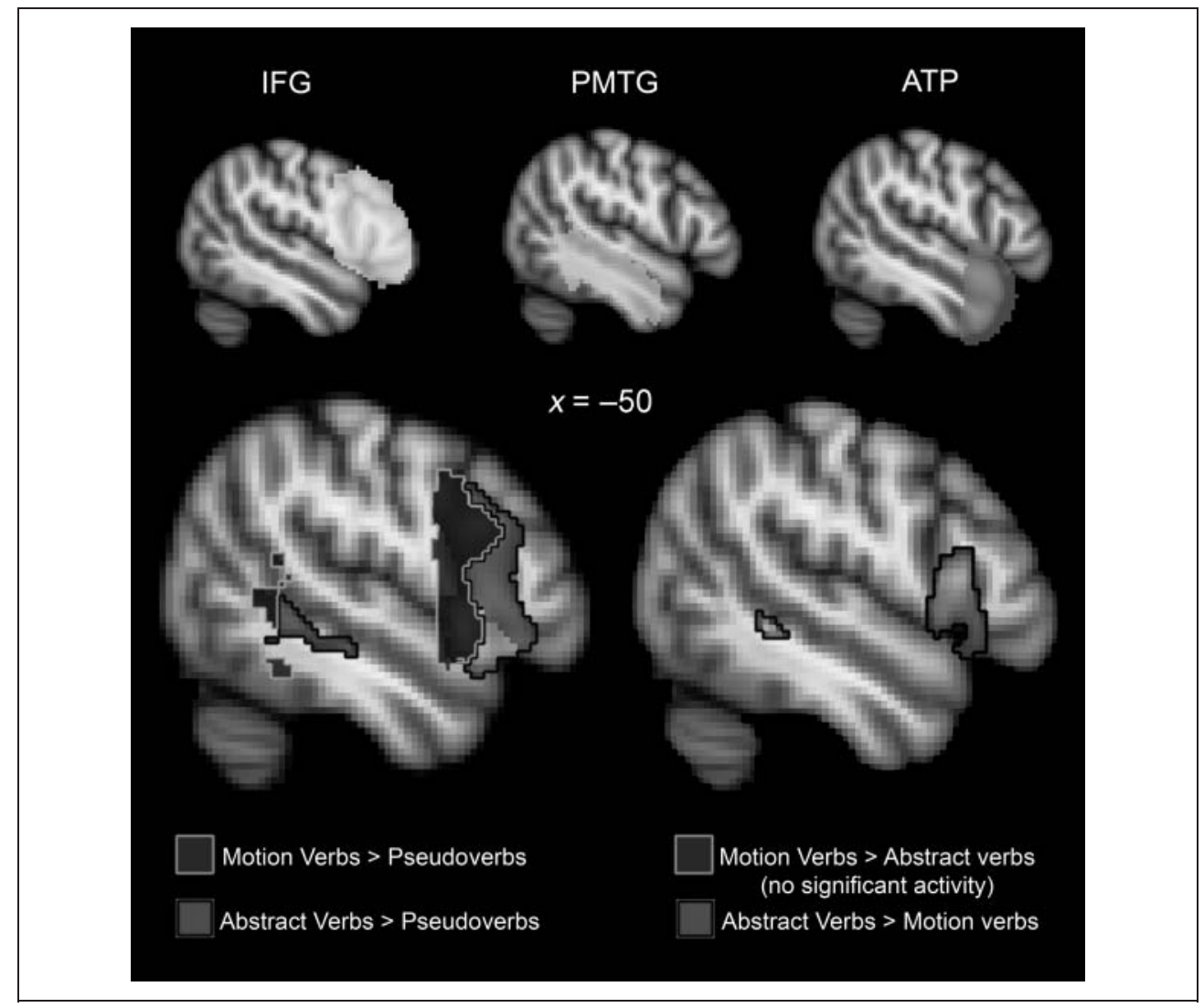

that were computed at the first-level analysis were computed at the group level for each ROI. Notice that these regions appear larger than the corresponding underlying structure on the MNI brain (e.g., the LIFG appears to extend beyond the precentral sulcus and the posterior middle temporal lobe mask includes most of the middle temporal lobe). This is because the atlas' regions are obtained by superimposing manually labeled individual brains, whose regions do not always align with those of the standard MIN brain due to anatomical individual differences. In any case, the large extent of these regions guarantees that all relevant activations are included in the analyses, although it may reduce statistical power.

\section{RESULTS}

\section{Overall Group Activations}

Table 2 reports the loci of activation for all contrasts. Comparisons of concrete verbs with the pseudoverb baseline revealed several bilateral clusters of activation, including the temporal-occipital, occipital fusiform, precentral, and angular gyri, as well as the central operculum $(~ p=$ .001 uncorrected). These areas have been previously associated with processing of motion and action stimuli in many studies (Kemmerer et al., 2007; Hauk et al., 2004; Beauchamp, Lee, Haxby, \& Martin, 2002; Kable et al., 2002). In contrast, various areas in the frontal and temporal cortices were significantly recruited in response to abstract verbs compared to pseudoverbs. These regions included the LIFG and RIFG, the anterior portion of the LPMTG, and the LATP. Activity near these regions has also been previously shown to be involved in processing abstract words (Sabsevitz et al., 2005; Noppeney \& Price, 2004; Grossman et al., 2002), converging on the interpretation that they play a role in processing abstract meaning. There were also some clusters shared by both concrete and abstract verbs that may indicate processing mechanisms common to both word types (e.g., precentral gyrus; see Vigneau et al., 2006).

\section{Region-of-Interest Analyses}

LIFG

Within this ROI, concrete verbs elicited greater activity than pseudoword controls at the edge of the precentral sulcus, whereas abstract verbs evoked more anterior activity, in the pars opercularis, posterior to the vertical ramus and extending into the Sylvian fissure $(p=.05$, corrected). The contrast between all abstract and all concrete words was also significant in this region (corrected $p=.05$ ). This significant cluster is located around the vertical and horizontal ramus (Figure 1). In comparisons made directly across the verb subcategories that were matched for argument structure complexity, transitive emotion verbs showed greater activity than transitive motion verbs (corrected $p=$ .05 ; Figure 2 , Table 3 ). Overall, the LIFG was differentially 
Table 2. Loci of Peak Voxels Obtained for the Indicated Contrasts in an Uncorrected $(p=.001)$ Whole-brain Analysis

\begin{tabular}{|c|c|c|c|c|c|}
\hline \multirow[b]{2}{*}{ Contrast } & \multirow[b]{2}{*}{ Activation Loci } & \multicolumn{3}{|c|}{ Coordinates (MNI) } & \multirow[b]{2}{*}{$Z$} \\
\hline & & $x$ & $y$ & $z$ & \\
\hline \multirow[t]{7}{*}{ Motion $>$ Pseudoverbs } & Bilateral precentral gyrus & -44 & -2 & -32 & 6.07 \\
\hline & Bilateral fusiform gyrus & 44 & -68 & -20 & 5.78 \\
\hline & Bilateral paracingulate cortex & -6 & 14 & 40 & 4.9 \\
\hline & Bilateral lateral occipital cortex & 34 & -70 & 46 & 4.86 \\
\hline & Left central opercular gyrus & -52 & 6 & -2 & 4.83 \\
\hline & Bilateral middle temporal gyrus & 52 & -40 & -2 & 4.71 \\
\hline & Right orbital cortex & 32 & 28 & 2 & 4.69 \\
\hline \multirow{11}{*}{ Abstract $>$ Pseudoverbs } & Right middle temporal gyrus & 50 & -42 & -2 & 5.89 \\
\hline & Left precentral gyrus & -44 & 0 & 32 & 5.85 \\
\hline & Bilateral fusiform gyrus & -24 & -88 & -12 & 5.48 \\
\hline & Bilateral inferior frontal gyrus & 54 & 12 & 24 & 5.11 \\
\hline & Bilateral paracingulate gyrus & -6 & 12 & 44 & 5.02 \\
\hline & Right lateral occipital cortex & 30 & -70 & 46 & 5.02 \\
\hline & Right insular cortex & 30 & 24 & -2 & 4.65 \\
\hline & Left middle temporal gyrus & -52 & -44 & -2 & 4.61 \\
\hline & Left superior parietal cortex & -36 & -56 & 56 & 4.5 \\
\hline & Left fusiform gyrus & -36 & -62 & -22 & 4.36 \\
\hline & Left anterior temporal pole & -52 & 8 & -4 & 4.3 \\
\hline \multirow[t]{3}{*}{ Motion Verbs > Abstract Verbs } & Left lateral occipital cortex & -40 & -80 & 22 & 3.6 \\
\hline & Left fusiform gyrus & -34 & -40 & -24 & 3.82 \\
\hline & Right lingual gyrus & 10 & -54 & 4 & 3.57 \\
\hline \multirow[t]{4}{*}{ Abstract Verbs $>$ Motion Verbs } & Bilateral inferior frontal gyrus & -54 & 18 & 12 & 3.52 \\
\hline & Left middle temporal gyrus & -60 & -44 & -4 & 3.95 \\
\hline & Left superior frontal gyrus & -14 & 24 & 56 & 3.3 \\
\hline & Bilateral frontal pole & -14 & 56 & 26 & 3.23 \\
\hline \multirow[t]{2}{*}{ Transitive Motion $>$ Transitive Emotion } & Left fusiform gyrus & -34 & -40 & -26 & 3.55 \\
\hline & Left lateral occipital cortex & -38 & -82 & 26 & 3.12 \\
\hline \multirow[t]{5}{*}{ Transitive Emotion $>$ Transitive Motion } & Left middle temporal gyrus & -60 & -44 & -4 & 4.24 \\
\hline & Left inferior frontal gyrus & -54 & 18 & 12 & 4.09 \\
\hline & Left inferior temporal gyrus & -44 & -4 & -30 & 3.96 \\
\hline & Right lingual gyrus & 10 & -54 & 2 & 3.55 \\
\hline & Left precentral gyrus & -42 & 4 & 36 & 3.46 \\
\hline
\end{tabular}

sensitive to all contrasts comparing abstract versus concrete semantics.

\section{RIFG}

In addition to contrasts relative to pseudowords, which parallel those in the LIFG, abstract words also show higher activation than concrete words in this region: Both the abstract versus concrete contrast and the transitive emotion versus transitive motion verb contrast (see Table 3 and Figures 1 and 2) were significant in a large cluster of voxels located in the pars opercularis near the vertical ramus $(p=$ .05 , corrected). This thus indicates that abstract verbs do engage the RIFG more strongly, compared to concrete 
Figure 2. Activations in different sagittal slices corresponding to the contrast between transitive emotion verbs and transitive motion verbs from the ROI analyses (cluster-corrected $Z=2.3$, $p=.05)$

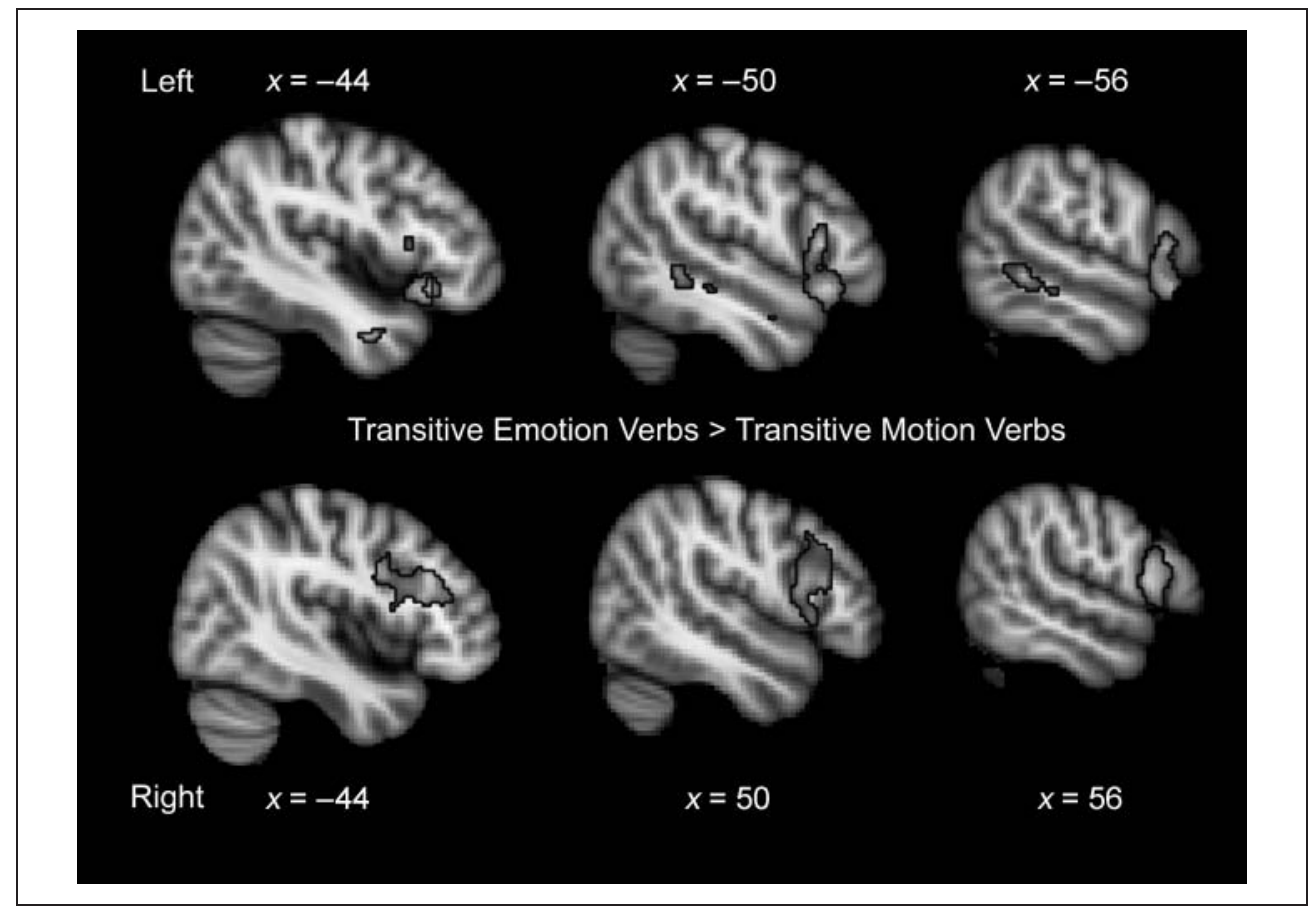

verbs, and is consistent with a retrieval demands view of abstract word processing.

\section{LATP}

All abstract verbs and all concrete verbs, compared to pseudoverbs ( $p=.05$ corrected), showed activity distributed along an axis running from posterior to anterior along the Sylvian fissure and the superior temporal gyrus, which, as shown in Figure 1, was part of the same cluster of activation as that of the LIFG ROI. Although activity overlaps across these comparisons, the clusters' centers are different (see Table 3). The contrast between transitive emotion verbs and transitive motion verbs also revealed significant activity in the vicinity of the Sylvian fissure as well as in the anterior region of the middle temporal gyrus ( $p=.05$, corrected) (Table 3, Figure 2 ). The contrast between all concrete and abstract words was not significant in this region.

\section{LPMTG}

Both abstract and concrete verbs, compared to pseudoverbs, showed activity in this region ( $p=.05$, corrected). As in the LIFG, the peak activations varied from posterior to anterior as abstract verbs showed greater activation than concrete verbs in the more anterior regions $(p=.05$, corrected). The contrast between transitive emotion and motion verbs (Figure 2), as well as the contrast between all abstract and concrete words (Figure 1), also yielded significant difference in the same cluster of voxels (see Table 3), indicating that in the more anterior region of PMTG, abstract verbs elicited higher activity than concrete verbs.

\section{DISCUSSION}

This study was completed to explore the role of the LIFG, LPMTG, and LATP, brain regions previously associated with semantic retrieval, in the processing of abstract compared to concrete verbs. The comparison between concrete verbs and pseudoverbs yielded a large distributed network of activations in bilateral regions, consistent with previous reports on action and motion word processing. However, motion verbs showed less activation than abstract verbs in the ROIs targeted here, suggesting little retrieval effort in processing motion verbs compared to abstract verbs. Yet different distributions of activations for each word type were apparent in the comparisons to pseudoverbs (see Table 3, Figure 1), indicating a progression from posterior to anterior in the LPMTG and LIFG, where concrete verbs show more posterior activity than abstract verbs. Abstract verbs, in contrast, showed a pattern of activity that was concentrated in left inferior frontal and temporal regions but, critically, also included the RIFG: All abstract verbs, including the subset of emotion verbs, showed higher activity than motion verbs near and around the vertical and/ or horizontal ramus in the LIFG and RIFG, in some cases, extending into the Sylvian fissure and the anterior superior temporal gyrus. Emotion verbs also elicited more activity than motion verbs in the anterior middle temporal lobe. Whereas all these left hemisphere areas are largely consistent with those previously reported in studies looking at abstract nouns with different tasks (Binder et al., 2005; Sabsevitz et al., 2005; Noppeney \& Price, 2004, see Tables 2-4), right IFG activations are not. Only one study (Perani, Cappa, et al., 1999) reported activity in a nearby region and this study, like the present one and unlike most other noun studies, included verbs in the stimuli. 
Table 3. Loci of Activation Peaks in Each ROI Analysis (Cluster-corrected $Z=2.3, p=.05$ )

\begin{tabular}{|c|c|c|c|c|c|}
\hline \multirow[b]{2}{*}{$R O I$} & \multirow[b]{2}{*}{ Contrast } & \multicolumn{3}{|c|}{ Coordinates (MNI) } & \multirow[b]{2}{*}{$Z$} \\
\hline & & $x$ & $y$ & $z$ & \\
\hline \multirow[t]{6}{*}{ Left inferior frontal gyrus } & Motion Verbs > Pseudoverbs & -46 & 2 & 34 & 5.55 \\
\hline & Abstract Verbs $>$ Pseudoverbs & -50 & 14 & 26 & 5.16 \\
\hline & Motion Verbs $>$ Abstract Verbs & \multicolumn{4}{|c|}{ no significant activations } \\
\hline & Abstract Verbs > Motion Verbs & -54 & 18 & 12 & 3.52 \\
\hline & Transitive Motion $>$ Transitive Emotion & \multicolumn{4}{|c|}{ no significant activations } \\
\hline & Transitive Emotion $>$ Transitive Motion & -54 & 18 & 12 & 4.09 \\
\hline \multirow[t]{6}{*}{ Right inferior frontal gyrus } & Motion Verbs > Pseudoverbs & 56 & 12 & 32 & 5.3 \\
\hline & Abstract Verbs > Pseudoverbs & 42 & 28 & 20 & 4.6 \\
\hline & Motion Verbs > Abstract Verbs & \multicolumn{4}{|c|}{ no significant activations } \\
\hline & Abstract Verbs > Motion Verbs & 62 & 16 & 12 & 3.21 \\
\hline & Transitive Motion $>$ Transitive Emotion & \multicolumn{4}{|c|}{ no significant activations } \\
\hline & Transitive Emotion $>$ Transitive Motion & 58 & 18 & 14 & 2.97 \\
\hline \multirow[t]{6}{*}{ Left posterior middle temporal gyrus } & Motion Verbs > Pseudoverbs & -56 & -52 & -2 & 4.1 \\
\hline & Abstract Verbs > Pseudoverbs & -54 & -44 & -2 & 5.03 \\
\hline & Motion Verbs > Abstract Verbs & \multicolumn{4}{|c|}{ no significant activations } \\
\hline & Abstract Verbs $>$ Motion Verbs & -52 & -42 & -4 & 4.09 \\
\hline & Transitive Motion $>$ Transitive Emotion & \multicolumn{4}{|c|}{ no significant activations } \\
\hline & Transitive Emotion $>$ Transitive Motion & -60 & -44 & -4 & 4.24 \\
\hline \multirow[t]{6}{*}{ Left anterior temporal pole } & Motion Verbs > Pseudoverbs & -50 & 6 & 0 & 4.81 \\
\hline & Abstract Verbs > Pseudoverbs & -50 & 10 & -4 & 4.42 \\
\hline & Motion Verbs > Abstract Verbs & \multicolumn{4}{|c|}{ no significant activations } \\
\hline & Abstract Verbs > Motion Verbs & \multicolumn{4}{|c|}{ no significant activations } \\
\hline & Transitive Motion $>$ Transitive Emotion & \multicolumn{4}{|c|}{ no significant activations } \\
\hline & Transitive Emotion $>$ Transitive Motion & -46 & 20 & -10 & 4.08 \\
\hline
\end{tabular}

Table 4. Peaks of Activation for the Abstract $>$ Concrete Contrast in the Present and Previous Studies

\begin{tabular}{|c|c|c|c|c|c|}
\hline \multirow[b]{2}{*}{ Study } & \multirow[b]{2}{*}{ Task } & \multirow[b]{2}{*}{ Region } & \multicolumn{3}{|c|}{ Coordinates (MNI) } \\
\hline & & & $x$ & $y$ & $z$ \\
\hline \multirow[t]{2}{*}{ This study } & \multirow[t]{2}{*}{ Silent reading } & Left inferior frontal gyrus & -54 & 18 & 12 \\
\hline & & Left middle temporal gyrus & -60 & -44 & -4 \\
\hline \multirow[t]{2}{*}{ Noppeney and Price (2004) } & \multirow[t]{2}{*}{ Semantic similarity judgment } & Left inferior frontal gyrus & -56 & 22 & -11 \\
\hline & & Left middle temporal gyrus & -62 & -44 & -7 \\
\hline \multirow[t]{2}{*}{ Sabsevitz et al. (2005) } & \multirow[t]{2}{*}{ Semantic similarity judgment } & Left inferior frontal gyrus & -44 & 23 & -10 \\
\hline & & Left middle temporal gyrus & -61 & -49 & 3 \\
\hline
\end{tabular}

Talairach and Tournoux (1988) coordinates reported in other studies where transformed into the MNI space with the icbm_fsl2tal tool (Lancaster et al., 2007). 
Thus, it is possible that abstract verbs engage more neural resources than nouns, given the more complex nature of their meaning (concerning, e.g., argument structure, thematic roles, the type of nouns that can co-occur with them, etc.).

Our finding of activity in the LIFG and RIFG is consistent with our hypothesis that abstract words engage more semantic processing and more difficult retrieval. Because some versions of dual-coding theory predict left-lateralized results for abstract words due to the expected activation of verbal information (Binder et al., 2005; Sabsevitz et al., 2005; Paivio, 1986), our finding that the RIFG is also recruited for abstract verb processing is not consistent with this left-lateralized interpretation of dual-coding theory. A large body of neuropsychological and imaging evidence also supports this view: The LIFG is selectively engaged in retrieving verb meanings (e.g., Shapiro \& Caramazza, 2001; Damasio \& Tranel, 1993) and has more generally been associated with regulatory functions such as maintaining, retrieving, and manipulating semantic information that need not be exclusively verbal (see Postle, 2006; Hagoort, 2005; Johnson-Frey, Newman-Norlund, \& Grafton, 2005; Thompson-Schill et al., 2005; Badre \& Wagner, 2002; Fuster, 2001; Miller \& Cohen, 2001; Duncan \& Owen, 2000; Passingham, Toni, \& Rushworth, 2000). In a meta-analysis of 129 language studies, Vigneau et al. (2006) conclude that clusters near those reported here are involved in controlled semantic retrieval and are modulated by the degree of control required. The RIFG, in turn, has also been proposed to aid the LIFG in its semantic functions, as the RIFG is recruited when more demanding retrieval and semantic selection processes take place. For example, Wagner et al. (2001) and Thompson-Schill et al. (1997) report more activity in the RIFG when stimulus words are weakly associated as opposed to strongly associated, and a meta-analysis of working memory studies highlights the role of the RIFG in such demanding conditions (Wagner \& Smith, 2003). Having weak associations to other concepts is a primary characteristic of abstract concepts (Schwanenflugel, 1991; de Groot, 1989). Therefore, it is not surprising that their retrieval requires more cognitive effort, and thus, right and left prefrontal engagement.

The processing view advocated here is also consistent with our findings in the temporal lobe, as this region is part of the network subserving modality-independent semantic processing together with the IFG. The anterior pole is often atrophied in semantic dementia patients and has been attributed several functions that resemble those of the LIFG, for example, amodal integration of semantics features, working memory, or semantic integration in sentence processing (Pobric et al., 2007; Jefferies \& LambonRalph, 2006; Vigneau et al., 2006; McClelland \& Rogers, 2003; Vandenberghe, Nobre, \& Price, 2002; Mummery et al., 1999). A recent study (Ghio \& Tettamanti, 2010) investigating functional integration patterns for concrete and abstract sentences has also reported reciprocal modulation between the areas reported here (PMTG, LIFG, and anterior temporal lobe), indicating that these regions are also involved in integrating semantic information across abstract words. Activity in posterior temporal regions, in turn, has long been associated with the storage and activation of action-related semantic information (Bedny et al., 2008; Gennari et al., 2007; Grossman et al., 2002; Kable et al., 2002; Martin \& Chao, 2001; Fiez, Raichel, Balota, Tallal, \& Petersen, 1996). Representations in this region are not modality specific, as they are active both for words and visual stimuli, and lesions in this region impair action understanding (Tranel et al., 2003; Kable et al., 2002; Chao, Haxby, \& Martin, 1999; Perani, Cappa, et al., 1999), but these representations may vary in specificity from posterior (more specific) to anterior (less specific) portions (Kable et al., 2002; Martin \& Chao, 2001). Moreover, posterior temporal regions are often coactivated with the LIFG in language processing studies (e.g., Tyler \& Marslen-Wilson, 2008) and very likely supply semantic information to the LIFG for integration or maintenance (Ghio \& Tettamanti, 2010; Gennari et al., 2007; Shtytov \& Pulvermüller, 2007; Pulvermuller, Shtyrov, \& Ilmoniemi, 2003; Thompson-Schill, 2003; Badre \& Wagner, 2002). Such observations are consistent with our findings in that distinct posterior temporal regions corresponding to different verb classes were coactivated with (partially distinct) areas of the LIFG, suggesting that semantic information retrieved in the PMTG was passed onto the LIFG through overlapping but distinct networks.

The view that abstract words' activations reflect more demanding retrieval or integration processes is compatible with findings showing that the processing of relatively abstract nouns, such as smell, is facilitated in the context of associate concepts, such as nose, more than is the processing of concrete nouns (Duñabeitia, Avilés, Afonso, Scheepers, \& Carreiras, 2009; Schwanenflugel \& Stowe, 1989; Kieras, 1978). Moreover, words that have a larger set of associates elicit less activation in the LIFG than words with fewer associates (Pexman, Hargreaves, Edwards, Henry, \& Goodyear, 2007a). Because concrete words tend to have more associates overall than abstract ones (de Groot, 1989), these studies suggest that, in the absence of facilitatory contexts, abstract words are less supported by associated information in their networks and require more effortful retrieval. When a facilitating context is available, its impact is larger on abstract words because their networks are less dense and tightly clustered so that activity spreads more rapidly through the network, as suggested by connectionist networks modeling abstract and concrete nouns as more or less richly interconnected features (Plaut \& Shallice, 1993; de Groot, 1989; Schwanenflugel \& Stowe, 1989).

It may be argued that the lack of difficulty effects in previous noun studies within the regions targeted here precludes a processing difficulty explanation (Sabsevitz et al., 2005; Noppeney \& Price, 2004). However, these studies used similarity or synonym decisions to word triads where the difficulty manipulation entailed longer semantic decisions. Therefore, the comparison between easy and hard 
conditions, which shows activity in the anterior cingulate and the insula, may not necessarily reflect the retrieval or integration of semantic properties that are associated with a word form. Rather, they may reflect difficult decisions, as shown in decision-making studies using different stimulus modalities (Kennerley, Walton, Behrens, Buckley, \& Rushworth, 2006; Heekeren, Marrett, Bandettini, \& Ungerleider, 2004). The fact that brain regions other than, say, the IFG, are associated with difficult decisions does not preclude that the retrieval of semantic information necessary to make a decision is also difficult and manifests in different regions from those engaged in decision-making. As it is often pointed out for assumptions of cognitive subtraction (D'Esposito, Zarahn, \& Aguirre, 1999), the decision component of a task could be independent from the semantic retrieval component, so that controlling for decision difficulty may not preclude differences in semantic retrieval demands.

In the present study, decisions had minimal impact in the observed activity because they only took place in $20 \%$ of the word trials after the word had already been read. Because the influence of catch trials was minimal in the averaged activity within and across blocks, the activations observed are very likely due to word reading, rather than decision-making. Yet, our behavioral results from the stimulus pretest do show differences in lexical decision times across abstract and concrete verbs. This finding, together with our neuroimaging results, suggests that either lexical decisions or simple word reading (with little influence of semantic decisions) depend on the process of activating the words' associated meaning, given the presentation of the word forms, because semantic retrieval is involved in both cases, independently of the task.

It may also be objected that the present results do not exclude a dual-coding interpretation because the involvement of the RIFG is only inconsistent with a strong leftlateralized version of this theory. It is indeed possible to claim, for example, that LIFG and RIFG are engaged in semantic retrieval, whereas the left temporal lobe provides the "verbal symbols" postulated by the dual-coding theory (Paivio, 1986). However, an increasing body of neuropsychological and imaging evidence (mentioned above) demonstrates that the temporal lobe stores conceptual information that is not exclusively verbal in nature: Posterior and anterior temporal lesions impair world knowledge, rather than language per se (Tranel et al., 2003; Mummery et al., 1999), and stimuli of different modalities activate the same temporal regions (e.g., Chao et al., 1999). Moreover, in Paivio's theory, abstract words within the verbal system have no direct links to the nonverbal system (Paivio, 1986, p. 67). Their referential connections to the world, if any, would depend exclusively on indirect associations through other words that may have nonverbal content. However, some abstract words (like our emotion verbs) have nonverbal emotional content (a possibility envisaged by Paivio), and several studies have demonstrated that abstract words do elicit nonverbal images (Barsalou \& Wiemer-Hastings,
2005; Wiemer-Hastings \& Xu, 2005). The evidence is, therefore, more in line with a view in which abstract words do have direct connections to experience, rather than being solely verbal in nature or only indirectly connected to it.

The processing view advocated here allows for connection to experience, and thus, is compatible with theories of distributed semantic representations such as those proposed by embodied approaches (e.g., Ghio \& Tettamanti, 2010; Pexman et al., 2007b; Barsalou \& Wiemer-Hastings, 2005; Wiemer-Hastings \& Xu, 2005). Surely, over the course of our lives, words become associated with all sorts of verbal and nonverbal experiences, which are likely to be distributed across the cortex. Experiences that become frequently associated with a word give rise to the pattern of activity elicited by the word (the word's network) when processed (Pulvermüller, 2005). Therefore, it may well be that abstract verbs are associated with distributed networks. However, the strength of their associations with correlated past experiences is likely to be weaker than that of concrete words because concrete words, individually and as a class, have very consistent and frequent co-occurring properties (e.g., motor associations), whereas abstract words have fewer associates and appear in more diverse contexts and have more diverse associated knowledge. This property of abstract word representation may explain why abstract words' networks often have less focalized activity in specific sensory-motor regions compared to concrete words, and it may explain why the only activity that was detected in this and in other fMRI studies corresponded to more demanding retrieval processes, rather than to stored semantic representations.

\section{Conclusions}

The present study investigated the processing of abstract verbs compared to concrete verbs. Results indicated that concrete and abstract activation networks (in comparison with activation elicited by pseudoverbs) were partially distinct, with concrete verbs eliciting more posterior activity. Moreover, abstract verbs elicited stronger activity in inferior frontal, anterior temporal, and posterior temporal regions. Compared to previous findings on nouns, verbs appear to more strongly engage the RIFG, which has been claimed to aid difficult retrieval. Together with available evidence demonstrating that the regions activated here store or process modality-independent semantic information, our pattern of results suggests that abstract verbs impose greater demands on semantic retrieval or semantic property integration due to the nature of their semantic networks, which are less consistent and more diverse across stimulus items, resulting in less focalized, difficult to detect, distributed activity. We therefore suggest that the network of regions activated in abstract verb processing is unlikely to reflect the activation of verbal codes or sentential context, as claimed in previous studies. The results can, 
nevertheless, be made consistent with distributed views of semantic memory, as distributed semantic networks may coexist with more difficult retrieval demands.

\section{Acknowledgments}

This research was funded by Grant MEC-SEJ2006-06712 from the Spanish Government. The first author was supported by a grant from the Gobierno del Principado de Asturias (Plan de Ciencia, Tecnología e Innovación de Asturias 2006-2009). Fernando Cuetos and Robert Davies were supported by the Marie Curie foundation grant (MRTN-CT-2004-512141) during the preparation of this report. We thank the staff at the York Neuroimaging Centre for support in conducting the study.

Reprint requests should be sent to Javier Rodríguez-Ferreiro, Facultat de Psicologia, Departament de Psicologia Bàsica, Edifici Ponent, Passeig de la Vall d'Hebron, 171, 08035, Barcelona, Spain, or via e-mail: rodriguezferreiro@ub.edu.

\section{REFERENCES}

Alameda, J. R., \& Cuetos, F. (1995). Diccionario de frecuencias de las unidades lingüísticas del castellano. Oviedo: Servicio de Publicaciones de la Universidad de Oviedo.

Audet, C., \& Burgees, C. (1999). Using a high-dimensional memory model to evaluate the properties of abstract and concrete words. In M. Hahn \& S. C. Stoness (Eds.), Proceedings of the Cognitive Science Society (pp. 37-42). Mahwah, NJ: Erlbaum.

Badre, D., \& Wagner, A. D. (2002). Semantic retrieval, mnemonic control, and prefrontal cortex. Behavioral and Cognitive Neuroscience Reviews, 1, 206-218.

Barsalou, L. W. (1999). Perceptual symbol system. Behavioral and Brain Sciences, 22, 577-609.

Barsalou, L. W. (2008). Grounded cognition. Annual Review of Psychology, 59, 617-645.

Barsalou, L. W., \& Wiemer-Hastings, K. (2005). Situating abstract concepts. In D. Pechner \& R. Zwaan (Eds.), Grounding cognition: The role of perception and action in memory, language and thought (pp. 129-163). New York: Cambridge University Press.

Beauchamp, M. S., Lee, K. E., Haxby, J. V., \& Martin, A. (2002). Parallel visual motion processing streams for manipulable objects and human movements. Neuron, 34, 149-159.

Beckmann, C., Jenkinson, M., \& Smith, S. M. (2003). General multi-level linear modelling for group analysis in FMRI. Neuroimage, 20, 1052-1063.

Bedny, M., Caramazza, A., Grossman, E., Pascual-Leone, A., \& Saxe, R. (2008). Concepts are more than percepts: The case of action verbs. Journal of Neuroscience, 28, $11347-11353$

Bedny, M., \& Thomson-Schill, S. L. (2006). Neuroanatomically separable effects of imageability and grammatical class during single-word comprehension. Brain and Language, 98, 127-139.

Binder, J. R., Westbury, C. F., McKiernan, K. A., Possing, E. T., \& Medler, D. A. (2005). Distinct brain systems for processing concrete and abstract concepts (Vol. 17, pp. 905-917). Cambridge, MA: MIT Press.

Chao, L. L., Haxby, J. V., \& Martin, A. (1999). Attribute-based neural substrates in temporal cortex for perceiving and knowing about objects. Nature Neuroscience, 2, 913-919.

Damasio, A. R., \& Tranel, D. (1993). Nouns and verbs are retrieved with differently distributed neural systems. Proceedings of the National Academy of Sciences, 90, 4957-4960.
Davis, C. J., \& Perea, M. (2005). BuscaPalabras: A program for deriving orthographic and phonological neighbourhood statistics and other psycholinguistic indices in Spanish. Behavior Research Methods, 37, 665-671.

de Groot, A. M. B. (1989). Representational aspects of word imageability and word frequency as assessed through word association. Journal of Experimental Psychology: Learning, Memory, and Cognition, 15, 824-845.

D'Esposito, M., Detre, J. A., Aguirre, G. K., Stallcup, M., Alsop, D. C., Tippet, L. J., et al. (1997). A functional MRI study of mental image generation. Neuropsychologia, 35, $725-730$.

D’Esposito, M., Zarahn, E., \& Aguirre, G. K. (1999). Event-related functional MRI: Implications for cognitive psychology. Psychological Bulletin, 125, 155-164.

Duñabeitia, J. A., Avilés, A., Afonso, O., Scheepers, C., \& Carreiras, M. (2009). Qualitative differences in the representation of abstract versus concrete words: Evidence from the visual-world paradigm. Cognition, 110, 284-292.

Duncan, J., \& Owen, A. M. (2000). Common regions of the human frontal lobe recruited by diverse cognitive demands. Trends in Neurosciences, 23, 475-483.

Fiez, J. A., Raichel, M. E., Balota, A. D., Tallal, P., \& Petersen, S. (1996). PET activation of posterior temporal regions during auditory word presentation and verb generation. Cerebral Cortex, 6, 1-10.

Fuster, J. M. (2001). The prefrontal cortex-An update: Time is of the essence. Neuron, 30, 319-333.

Gennari, S. P., MacDonald, M. C., Postle, B. R., \& Seidenberg, M. S. (2007). Context-dependent interpretation of words: Evidence for interactive neural processes. Neuroimage, 35, 1278-1286.

Ghio, M., \& Tettamanti, M. (2010). Semantic domain-specific functional integration for action-related vs. abstract concepts. Brain and Language, 112, 223-232.

Grezes, J., \& Decety, J. (2001). Functional anatomy of execution, mental simulation, observation, and verb generation of actions: A meta-analysis. Human Brain Mapping, 12, 1-19.

Grossman, M., Koenig, P., DeVita, C., Glosser, G., Alsop, D., Detre, J., et al. (2002). Neural representation of verb meaning: An fMRI study. Human Brain Mapping, 15, 124-134.

Hagoort, P. (2005). On Broca, brain, and binding: A new framework. Trends in Cognitive Sciences, 9, 416-423.

Hauk, O., Johnsrude, I., \& Pulvermüller, F. (2004). Somatotopic representations of action words in human motor and premotor cortex. Neuron, 41, 301-307.

Heekeren, H. R., Marrett, S., Bandettini, P. A., \& Ungerleider, L. G. (2004). A general mechanism for perceptual decision-making in the human brain. Nature, 431, 859-861.

Jefferies, E., \& Lambon-Ralph, M. A. (2006). Semantic impairment in stroke aphasia versus semantic dementia: A case-series comparison. Brain, 129, 2132-2147.

Jenkinson, M., Bannister, P., Brady, M., \& Smith, S. M. (2002). Improved optimisation for the robust and accurate linear registration and motion correction of brain images. Neuroimage, 17, 825-841.

Jenkinson, M., \& Smith, S. M. (2001). A global optimisation method for robust affine registration of brain images. Medical Image Analysis, 5, 143-156.

Johnson-Frey, S. H., Newman-Norlund, R., \& Grafton, S. T. (2005). A distributed left hemisphere network active during planning of everyday tool use skills. Cerebral Cortex, 15, 681-695.

Jones, G. V. (1985). Deep dyslexia, imageability, and ease of predication. Brain and Language, 24, 1-19.

Kable, J. W., Kan, I. P., Wilson, A., Thomson-Schill, S. L., \& Chatterjee, A. (2005). Conceptual representations of 
action in the lateral temporal cortex. Journal of Cognitive Neuroscience, 17, 1855-1870.

Kable, J. W., Lease-Spellmeyer, J., \& Chatterjee, A. (2002). Neural substrates of action event knowledge. Journal of Cognitive Neuroscience, 14, 795-805.

Kemmerer, D., González-Castillo, J., Talavage, T., Patterson, S., \& Wiley, C. (2007). Neuroanatomical distribution of five semantic components of verbs: Evidence from fMRI. Brain and Language, 107, 16-43.

Kennerley, S. W., Walton, M. E., Behrens, T. E., Buckley, M. J., \& Rushworth, M. F. (2006). Optimal decision making and the anterior cingulate cortex. Nature Neuroscience, 9, 940-947.

Kiehl, K. A., Liddle, P. F., Smith, A. M., Mendrek, A., Forster, B. B., \& Hare, R. D. (1999). Neural pathways involved in the processing of concrete and abstract words. Human Brain Mapping, 7, 225-233.

Kieras, D. (1978). Beyond pictures and words: Alternative information-processing models for imagery effects in verbal memory. Psychological Bulletin, 85, 532-554.

Lancaster, J. L., Tordesillas-Gutiérrez, D., Martinez, M., Salinas, F., Evans, A., Zilles, K., et al. (2007). Bias between MNI and Talairach coordinates analyzed using the ICBM-152 brain template. Human Brain Mapping, 28, 1149-1205.

Martin, A., \& Chao, L. L. (2001). Semantic memory and the brain: Structure and processes. Current Opinion in Neurobiology, 11, 194-201.

McClelland, J. L., \& Rogers, T. T. (2003). The parallel distributed processing approach to semantic cognition. Nature Reviews Neuroscience, 4, 310-322.

Mellet, E., Tzourio, N., Denis, M., \& Mazoyer, B. (1998). Cortical anatomy of mental imagery of concrete nouns based on their dictionary definition. NeuroReport, 1998, 803-808.

Miller, E. K., \& Cohen, J. D. (2001). An integrative theory of prefrontal cortex function. Annual Review of Neuroscience, 24, 167-202.

Mummery, C. J., Patterson, K., Wise, R. J. S., Vandenbergh, R., Price, C. J., \& Hodges, J. R. (1999). Disrupted temporal lobe connections in semantic dementia. Brain, 122, 61-73.

Noppeney, U., \& Price, C. J. (2004). Retrieval of abstract semantics. Neuroimage, 22, 164-170.

Paivio, A. (1986). Mental representation; Dual-coding bypothesis. Oxford: Oxford University Press.

Paivio, A. (1991). Dual coding theory: Retrospect and current status. Canadian Journal of Psychology, 45, 255-287.

Passingham, R. E., Toni, I., \& Rushworth, M. F. (2000). Specialisation within the prefrontal cortex: The ventral prefrontal cortex and associative learning. Experimental Brain Research, 133, 103-113.

Perani, D., Cappa, S. F., Schnur, T., Tettamanti, M., Collina, S., Rosa, M. M., et al. (1999). The neural correlates of verb and noun processing: A PET study. Brain, 122, 2337.

Perani, D., Schnur, T., Tettamanti, M., Gorno-Tempini, M., Cappa, S. F., \& Fzio, F. (1999). Word and picture matching: A PET study of semantic category effects. Neuropsychologia, 37, 293-306.

Pexman, P. M., Hargreaves, I. S., Edwards, J. D., Henry, L. C., \& Goodyear, B. G. (2007a). The neural consequences of semantic richness: When more comes to mind, less activation is observed. Psychological Science, 18, 401-406.

Pexman, P. M., Hargreaves, I. S., Edwards, J. D., Henry, L. C., \& Goodyear, B. G. (2007b). Neural correlates of concreteness in semantic categorization. Journal of Cognitive Neuroscience, 19, 1407-1419.

Plaut, D. C., \& Shallice, T. (1993). Deep dyslexia: A case study of connectionist neuropsychology. Cognitive Neuropsychology, $10,377-500$
Pobric, G., Jefferies, E., \& Lambon Ralph, M. A. (2007). Anterior temporal lobes mediate semantic representation: Mimicking semantic dementia by using rTMS in normal participants. Proceedings of the National Academy of Sciences, U.S.A., 104, 20137-20141.

Postle, B. R. (2006). Working memory as an emergent property of the mind and brain. Neuroscience, 139, 23-38.

Pulvermüller, F. (2005). Brain mechanisms linking language and action. Nature Reviews Neuroscience, 6, 576-582.

Pulvermuller, F., Shtyrov, Y., \& Ilmoniemi, R. (2003). Spatiotemporal dynamics of neural language processing: An MEG study using minimum-norm current estimates. Neuroimage, 20, 1020-1025.

Rüschemeyer, S. A., Brass, M., \& Friederici, A. D. (2007). Comprehending prehending: Neural correlates of processing verbs with motor stems. Journal of Cognitive Neuroscience, 19, 855-865.

Sabsevitz, D. S., Medler, D. A., Seidenberg, M., \& Binder, J. R. (2005). Modulation of the semantic system by word imageability. Neuroimage, 27, 188-200.

Sadoski, M., \& Paivio, A. (2004). A dual coding theoretical model of reading. In R. B. Ruddell \& N. J. Unrau (Eds.), Theoretical models and processes of reading (pp. 1329-1362). Newark, DE: International Reading Association.

Schwanenflugel, P. J. (1991). Why are abstract concepts hard to understand? In P. Schwanenflugel (Ed.), The psychology of word meaning (pp. 223-250). Hillsdale, NJ: Erlbaum.

Schwanenflugel, P. J., \& Stowe, R. W. (1989). Context availability and the processing of abstract and concrete words in sentences. Reading Research Quarterly, 24, 114-126.

Shapiro, K. A., \& Caramazza, A. (2001). Sometimes a noun is just a noun: Comments on Bird, Howard and Franklin (2000). Brain and Language, 76, 202-212.

Shapiro, K. A., Pascual-Leone, A., Mottaghy, F. M., Gangitano, M., \& Caramazza, A. (2001). Grammatical distinctions in the left frontal cortex. Journal of Cognitive Neuroscience, 13, 472-478.

Shapiro, L. P., Nagel, H. N., \& Levine, B. A. (1993). Preferences for a verb's complements and their use in sentence processing. Journal of Memory and Language, 32, 96-114.

Shtytov, Y., \& Pulvermüller, F. (2007). Early MEG activation dynamics in the left temporal and inferior frontal cortex reflect semantic context integration. Journal of Cognitive Neuroscience, 19, 1633-1642.

Talairach, J., \& Tournoux, P. (1988). Co-planar stereotaxic atlas of the buman brain. New York: Thieme.

Tettamanti, M., Buccino, G., Saccuman, M. C., Gallese, V., Danna, M., Scifo, P., et al. (2005). Listening to action-related sentences activates fronto-parietal motor circuits. Journal of Cognitive Neuroscience, 17, 273-281.

Thompson-Schill, S. L. (2003). Neuroimaging studies of semantic memory: Inferring "how" from "where." Neuropsychologia, 41, 280-292.

Thompson-Schill, S. L., Bedny, M., \& Goldberg, R. F. (2005). The frontal lobes and the regulation of mental activity. Current Opinion in Neurobiology, 15, 219-224.

Thompson-Schill, S. L., D’Esposito, M., Aguirre, G. K., \& Farah, M. J. (1997). Role of left inferior prefrontal cortex in retrieval of semantic knowledge: A reevaluation. Proceedings of the National Academy of Sciences, U.S.A., 94, 14792-14797.

Tranel, D., Kemmerer, D., Adolphs, R., Damasio, H., \& Damasio, A. (2003). Neural correlates of conceptual knowledge for actions. Cognitive Neuropsychology, 20, 409-432.

Tyler, L. K., \& Marslen-Wilson, W. (2008). Fronto-temporal brain systems supporting spoken language comprehension. Philosophical Transactions of the Royal Society of London, Series B, Biological Sciences, 363, 1037-1054.

Tyler, L. K., Stamatakis, E. A., Dick, E., Bright, P., Fletcher, P., \& Moss, H. M. (2003). Objects and their actions: Evidence 
for a neurally distributed semantic system. Neuroimage, $18,542-557$.

Vandenberghe, R., Nobre, A. C., \& Price, C. J. (2002). The response of left temporal cortex to sentences. Journal of Cognitive Neuroscience, 14, 550-560.

Vigneau, M., Beaucousin, V., Herve, P. Y., Duffau, H., Crivello, F., Houde, O., et al. (2006). Meta-analyzing left hemisphere language areas: Phonology, semantics, and sentence processing. Neuroimage, 30, 1414-1432.

Wagner, A. D., Paré-Blagoev, E. J., Clark, J., \& Poldrack, R. A. (2001). Recovering meaning: Left prefrontal cortex guides controlled semantic retrieval. Neuron, 31, 329-338.
Wagner, T. D., \& Smith, E. E. (2003). Neuroimaging studies of working memory: A meta-analysis. Cognitive, Affective and Behavioral Neuroscience, 3, 255-274.

Wiemer-Hastings, K., \& Xu, X. (2005). Content differences for abstract and concrete concepts. Cognitive Science: A Multidisciplinary Journal, 29, 719-736.

Woolrich, M. W., Behrens, T. E. J., Beckmann, C. F., Jenkinson, M., \& Smith, S. M. (2004). Multi-level linear modelling for FMRI group analysis using Bayesian inference. Neuroimage, 21, $1732-1747$.

Woolrich, M. W., Ripley, B. D., Brady, J. M., \& Smith, S. M. T. (2001). Temporal autocorrelation in univariate linear modelling of FMRI data. Neuroimage, 14, 1370-1386. 
Copyright of Journal of Cognitive Neuroscience is the property of MIT Press and its content may not be copied or emailed to multiple sites or posted to a listserv without the copyright holder's express written permission. However, users may print, download, or email articles for individual use. 